\title{
Comparing the effects of vaginal misoprostol, laminaria, and extra amniotic saline infusion on cervical ripening and induction of labor
}

\author{
Zohreh Tabasi, MD', Elaheh Mesdaghinia, MD¹, Masoumeh Abedzadeh-Kalahroudi, PhD², Mojtaba Sehat, \\ MD, PhD ${ }^{3}$, Aida Panahandeh, MD' \\ ${ }^{1}$ Department of Obstetrics and Gynecology, ${ }^{2}$ Trauma Nursing Research Center, ${ }^{3}$ Trauma Research Center, Kashan University of Medical Sciences, \\ Kashan, Iran
}

\section{Objective}

This study aimed to compare the effects of vaginal misoprostol, laminaria, and extra-amniotic saline infusion (EASI) on cervical ripening.

\section{Methods}

This randomized controlled trial was conducted on 195 women with singleton pregnancies and unripe cervices. Participants were randomly allocated to 3,65-person groups: a misoprostol, a laminaria, and an EASI group. The interventions in the misoprostol, laminaria, and EASI groups included a single $25-\mu \mathrm{g}$ vaginal misoprostol suppository, an intracervical laminaria, and a transcervical Foley catheter, respectively. The groups were compared with each other regarding time intervals from labor induction to labor active phase and delivery, cervical dilation, Bishop scores 6 hours after induction, delivery type, length of hospital stay, and complications.

Results

There were no significant differences among the groups regarding maternal ages, gestational ages, body mass indices, baseline cervical dilations, and Bishop scores (P>0.05). Six hours after induction, the Bishop score and cervical dilation were significantly greater in the EASI group than in the other 2 groups $(P<0.001)$. Moreover, time intervals from labor induction to labor active phase and delivery in the EASI group were significantly short $(P<0.001)$. The rates of cesarean section, fetal distress, placental abruption, and meconium staining in the misoprostol group were significantly high $(P<0.05)$, and the length of hospital stay in the EASI group was significantly short $(P<0.001)$.

\section{Conclusion}

EASI is a safer and more effective method for cervical ripening. Considering its inexpensiveness, easy accessibility, and greater effectiveness, EASI is recommended for cervical ripening.

Trial Registration

Iranian Center for Clinical Trials Identifier: IRCT20170513033941N39

Keywords: Misoprostol; Cervical Ripening; Laminaria; Labor

\section{Introduction}

Before spontaneous labor, induction of labor may be indicated if the advantages of pregnancy termination outweigh the advantages of its continuation [1]. The rate of labor induction in the United States has increased by 2.5 times from $9.5 \%$ in 1990 to $25.7 \%$ in 2017 [2]. Some indications

Received: 2019.09.04. Revised: 2019.10.20. Accepted: 2019.10.31. Corresponding author: Masoumeh Abedzadeh-Kalahroudi, PhD Trauma Nursing Research Center, Kashan University of Medical Sciences, Ravand Road, Kashan University of medical Sciences, Kashan 87137-81147, Iran

E-mail: Abedzadeh@kaums.ac.ir https://orcid.org/0000-0002-7318-6294

Articles published in Obstet Gynecol Sci are open-access, distributed under the terms of the Creative Commons Attribution Non-Commercial License (http://creativecommons. org/licenses/by-nc/3.0/) which permits unrestricted non-commercial use, distribution, and reproduction in any medium, provided the original work is properly cited.

Copyright $\odot 2020$ Korean Society of Obstetrics and Gynecology 


\section{Obstetrics \& Gynecology Science}

Vol. 63, No. 3, 2020

for labor induction include severe pre-eclampsia, post-term pregnancy, and premature rupture of membranes [3].

Cervical status and its ripening are important factors for successful induction of labor. There are different pharmacological and mechanical methods for cervical ripening. The best cervical ripening method is selected based on its effectiveness and safety and also patient's medical and obstetric history and clinical characteristics [4]. Administration of misoprostol, or prostaglandin $\mathrm{E} 1$, is one of the pharmacological methods for cervical ripening. It is used either vaginally or orally. It affects the amount of cervical collagen production, increases the degradation of the collagen matrix in the cervix, and thereby, softens and ripens the cervix. Moreover, it induces uterine contractions. However, misoprostol, particularly at doses of more than $50 \mu \mathrm{g}$, increases the need for cesarean sections and the risks of hyperstimulation, uterine ruptures, neonatal intensive care unit admissions, and meconium staining [5]. Because of the serious adverse effects of pharmacological methods for cervical ripening, mechanical methods have recently received great attention and extensive research has been done on their effectiveness [6]. These methods include extra-amniotic saline infusions (EASI) via transcervical catheters, hygroscopic dilators such as laminaria, and membrane stripping. Transcervical catheters are used either with or without saline infusions. Laminaria is a synthetic type of sea algae which induces cervical dilation by absorbing water from the cervix and gradually increasing in thickness [3].

To date, different studies have been conducted on the effects and safety of pharmacological and mechanical methods for cervical ripening. Yet, controversies still exist over the best cervical ripening method [7]. A study reported that the time interval from labor induction to the labor active phase in the EASI group was shorter than that in the laminaria group, and there were no significant differences in complications between these 2 methods [8]. One study indicated that misoprostol was more effective than laminaria in increasing the Bishop scores and shortening labor durations, though cesarean section rates in the misoprostol group were greater [9]. However, another study found that although this interval was shorter in the laminaria group than in the misoprostol group, the difference was not statistically significant. Moreover, the rates of cesarean sections and uterine hyperstimulation were greater in the misoprostol group [10]. A comparative study into the effects of EASI and misoprostol found that induction-labor time intervals and Bishop score changes were significantly lower in the misoprostol group than in the EASI group, while uterine hyperactivity and ruptures were significantly more prevalent in the misoprostol group. That study concluded that $100 \mu \mathrm{g}$ misoprostol suppositories were more effective than EASI catheters in cervical ripening and labor induction, but recommended further studies to determine safe doses of misoprostol [11]. A study concluded that when pregnancy termination was needed and the cervix was not ripe, vaginal misoprostol was more effective than EASI in shortening labor durations and reducing the need for cesarean sections [7]. The results of another study illustrated that there were no significant differences between the misoprostol and EASI groups regarding neonatal Apgar scores, meconium staining rates, cesarean section rates, and the time intervals between labor induction and labor active phases; however, the time interval between labor induction and delivery in the misoprostol group was significantly shorter than that in the EASI group [12]. However, a meta-analysis study reported no significant differences between misoprostol and EASI methods regarding cesarean section rates and the time intervals from labor induction to active labor phases and delivery [13].

These contradictory results of previous studies highlight the necessity of additional studies on the effects of pharmacological and mechanical cervical ripening methods. Moreover, none of the previous studies compared the effects of these 3 methods for cervical ripening. Therefore, the present study was conducted to compare the effects of vaginal misoprostol, laminaria, and EASI on cervical ripening and labor induction.

\section{Materials and methods}

\section{Participants}

This 3-group randomized controlled trial was conducted on 195 pregnant women who required cervical ripening and labor inductions. Participants were recruited in 2017-2018 from Shahid Beheshti and Shabihkhani hospitals, Kashan, Iran. Inclusion criteria were: a live fetus with cephalic presentation, a normal nonstress test, a gestational age of 37 weeks or more, fetal weight less than 4 kilograms, a Bishop score less than 5, no labor pains, and no contraindications for vaginal delivery. Participants were excluded if they 


\section{Obstetrics \& Gynecology Science}

Zohreh Tabasi, et al. Misoprostol, laminaria, and EASI on cervical ripening

developed fetal distress, thick meconium-stained amniotic fluid, fever, chorioamnionitis, or vaginal bleeding before intervention.

\section{Sample size}

Sample size was calculated using the results of a previous study [8] which reported that the time intervals between labor induction and delivery in the Foley catheter and laminaria groups were $10.3 \pm 4.35$ and $12.5 \pm 4.9$ hours, respectively. With a confidence level of $95 \%$ and a power of $80 \%$, the sample size was estimated to be 65 per group. Using an online random number generator and through permuted block randomization with a block size of 6, all 195 eligible pregnant women were randomly allocated to groups $A$ (misoprostol), B (laminaria), or C (EASI). Sequentially numbered opaque sealed envelopes were used for allocation concealment. The generated allocation sequence was written on cards and the cards were put into envelopes. For each new participant, 1 envelope was opened, and her group was determined based on the label on the card.

\section{Intervention}

Participants in the misoprostol group received a single $25-\mu \mathrm{g}$ vaginal misoprostol suppository (Cytotex, Searle, England). The suppository was placed in the posterior fornix of the vagina. In the laminaria group, the cervix was initially washed with povidone-iodine, and laminaria were placed inside using ring forceps. In the EASI group, a Foley catheter (size 22) was placed under sterile conditions in the uterus through the cervix and its balloon was filled with $40 \mathrm{~mL}$ of distilled water so that the balloon was located behind the internal orifice of the uterus. The other end of the catheter was connected to a $500 \mathrm{~mL}$ saline solution bottle, and the infusion rate was set at $40 \mathrm{~mL}$ per hour. The catheter was in place until spontaneously expelled from the uterus. All women in the 3 groups underwent vaginal examinations every 2 hours, and their Bishop scores were calculated. If any participant did not spontaneously enter the active phase of labor after 6 hours (i.e., did not have regular uterine contractions and progressive cervical changes), oxytocin was used for labor induction according to the hospital protocol, and the patient was

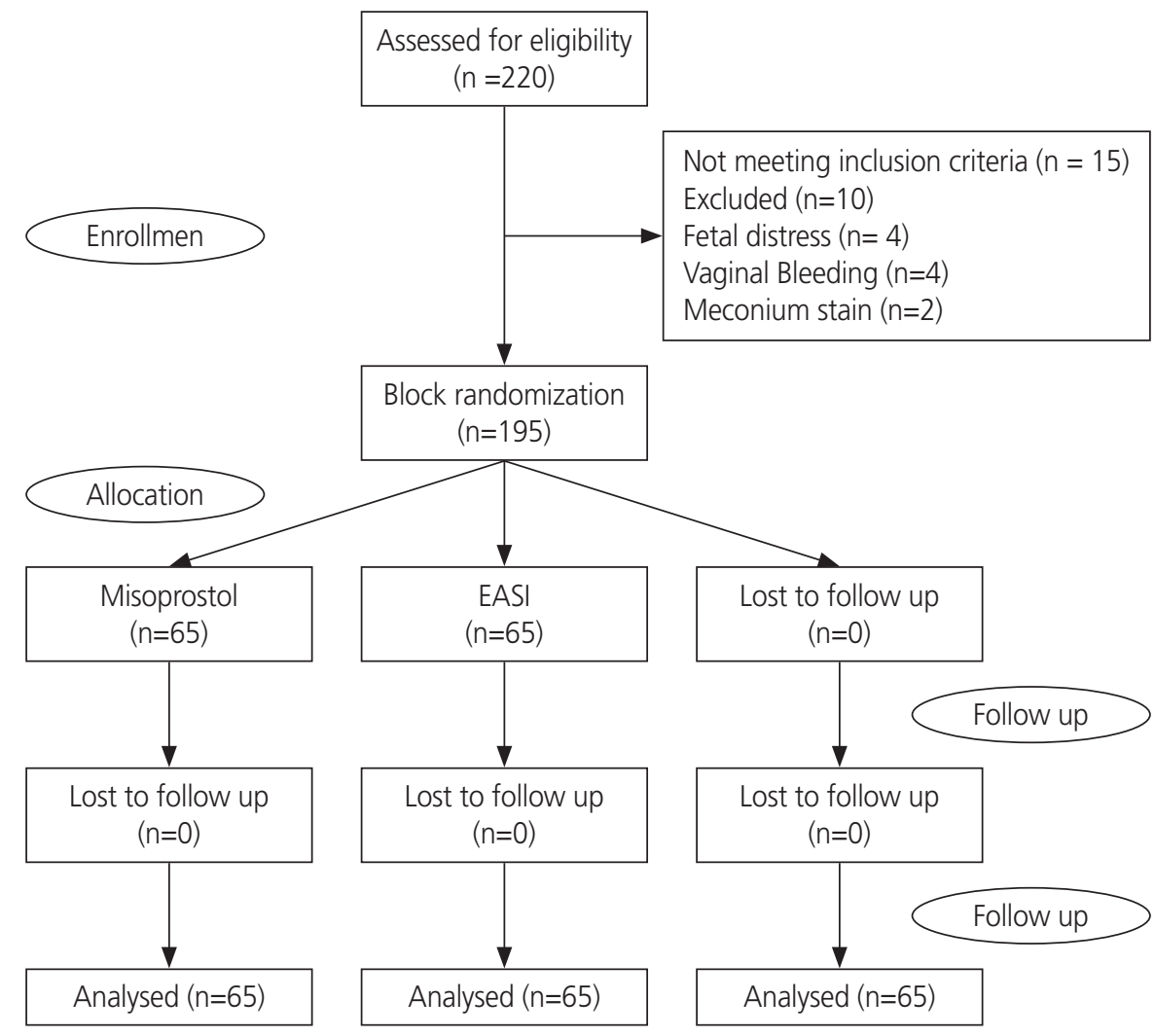

Fig. 1. Consort diagram. EASI, extra-amniotic saline infusion. 


\title{
Obstetrics \& Gynecology Science
}

\author{
Vol. 63 , No. 3, 2020
}

monitored until delivery. The 3 groups were also assessed for complications, including dilatation arrest, uterine hypertonicity, tachysystole (i.e., at least 5 uterine contractions per 10 minutes), placental abruption, meconium staining, and fetal distress.

A checklist was used to document study outcomes and included maternal age, gestational age, body mass index (BMI), baseline and 6-hour cervical dilation, Bishop scores, the time intervals from labor induction to labor active phase and delivery, route of delivery, and ripening method complications.

\section{Statistical data analysis}

Data analysis was performed using a SPSS program (version 16.0; SPSS Inc., Chicago, IL, USA). Within- and betweengroup comparisons regarding numerical variables were analyzed using the paired sample $t$-test and 1-way analysis of variance with post hoc analysis, respectively. Between-group comparisons regarding categorical variables were analyzed using the $\chi^{2}$ test. The level of significance was set at less than 0.05 .

\section{Results}

In total, 210 women were assessed for eligibility and 195 eligible women were studied in 3 groups (Fig. 1). Findings re- vealed no significant differences among the groups regarding participants' demographic and reproductive characteristics and causes of labor induction (Table 1).

There was no significant among-group difference with regard to the pretest mean of Bishop scores $(P=0.066)$. However, 6 hours after the intervention, the mean post-test Bishop scores among groups differed significantly from each other $(P<0.001)$. The results of the Tukey's post hoc test indicated that the mean of the posttest Bishop scores in the laminaria group was significantly less than those in the misoprostol and the EASI groups $(P<0.001)$, while there were no significant differences between the misoprostol and EASI groups $(P=0.13)$. Moreover, within group comparisons revealed that the means of the posttest Bishop scores in all 3 groups were significantly greater than their corresponding pretest values $(P<0.001$; Table 2).

There was no statistically significant difference among the groups regarding the pretest mean values of cervical dilation $(P=0.441)$, while the among group difference regarding the posttest mean value of cervical dilation was statistically significant $(P<0.001)$. The Tukey's post hoc test showed that the posttest mean value of cervical dilation in the EASI group was significantly greater than those in the other 2 groups, and the post-test mean value of cervical dilation in the misoprostol group was significantly greater than that in the laminaria group $(P<0.001)$. Within group comparisons also

Table 1. Comparison of demographic and reproductive characteristics among the 3 groups

\begin{tabular}{|c|c|c|c|c|}
\hline \multirow{2}{*}{ Variables } & \multicolumn{3}{|c|}{ Groups } & \multirow{2}{*}{$P$-value } \\
\hline & Misoprostol & Laminaria & EASI & \\
\hline Maternal age (range 15-40 yr) & $28.32 \pm 4.98$ & $27.29 \pm 6.05$ & $28.20 \pm 5.56$ & 0.513 \\
\hline Gestational age (range 37-41 wk) & $38.94 \pm 1.17$ & $38.52 \pm 2.12$ & $39.06 \pm 1.14$ & 0.118 \\
\hline $\mathrm{BMI}$ & $27.57 \pm 5.0$ & $26.48 \pm 6.37$ & $26.74 \pm 4.75$ & 0.490 \\
\hline \multicolumn{5}{|l|}{ Parity } \\
\hline Nullipara & $34(52.3)$ & $32(49.2)$ & $37(56.9)$ & 0.676 \\
\hline Multipara & $31(47.7)$ & $33(50.8)$ & $28(43.1)$ & \\
\hline \multicolumn{5}{|l|}{ Cause of induction } \\
\hline $\mathrm{PIH}$ & $25(38.4)$ & $22(33.8)$ & $20(30.7)$ & 0.860 \\
\hline Postdate & $30(46.2)$ & $32(49.2)$ & $31(47.7)$ & - \\
\hline Oligohydramnios & $5(7.7)$ & $4(6.2)$ & $4(6.2)$ & - \\
\hline $\begin{array}{l}\text { Maternal diseases (diabetes, chronic } \\
\text { hypertension...) }\end{array}$ & $5(7.7)$ & $7(10.8)$ & $10(15.4)$ & - \\
\hline
\end{tabular}

Data are shown as mean \pm standard deviation or number (\%).

BMI, body mass index; PIH, pregnancy-induced hypertension; EASI, extra-amniotic saline infusion. 


\section{Obstetrics \& Gynecology Science}

Zohreh Tabasi, et al. Misoprostol, laminaria, and EASI on cervical ripening

revealed that the posttest mean values of cervical dilation in all groups were significantly greater than their corresponding pretest values $(P<0.001$; Table 2$)$.

The groups also differed significantly from each other regarding the time intervals from labor induction to labor active phase and delivery $(P<0.001)$. The results of the Tukey's post hoc test revealed that the time interval in the EASI group was significantly shorter than that in the other 2 groups $(P<0.01)$. Moreover, the time interval in the misoprostol group was significantly shorter than that in the laminaria group $(P<0.01$; Table 2).

Among group differences regarding the lengths of hospital stays were statistically significant $(P<0.001)$. The Tukey's post hoc test showed that the length of hospital stay in the EASI group was significantly shorter than that in the other 2 groups; it was significantly shorter in the laminaria group than in the misoprostol group $(P<0.05)$.

The groups also significantly differed from each other regarding the routes of delivery $(P=0.026)$. Pairwise comparisons indicated that the rate of vaginal delivery in the EASI group (81.5\%) was significantly higher than that in the misoprostol group (60\%) ( $P=0.007)$. However, the differences between the misoprostol and laminaria groups and between the EASI and laminaria groups regarding the rates of vaginal deliveries were not significant ( $P>0.05$; Table 2 ).

There were significant differences among the groups regarding meconium staining, placental abruption, and fetal distress. The highest prevalence rates of meconium staining (16.9\%), placental abruption (15.4\%), and fetal distress $(21.5 \%)$ were observed in the misoprostol group (Table 3).

After adjusting for possible confounders, the analysis of covariance model showed that maternal age, gestational age,

Table 2. Among-group comparisons according to ripening outcomes

\begin{tabular}{|c|c|c|c|c|c|}
\hline \multirow{2}{*}{\multicolumn{2}{|c|}{ Variables }} & \multicolumn{3}{|c|}{ Groups } & \multirow{2}{*}{$P$-value } \\
\hline & & Misoprostol & Laminaria & EASI & \\
\hline \multirow[t]{2}{*}{ Bishop score } & Baseline & $2.42 \pm 0.61$ & $2.42 \pm 0.66$ & $2.65 \pm 0.67$ & 0.066 \\
\hline & 6 hours later & $6.25 \pm 1.59$ & $5.09 \pm 0.91$ & $6.74 \pm 1.71$ & $<0.001$ \\
\hline$P$-value & & $<0.001$ & $<0.001$ & $<0.001$ & \\
\hline \multirow[t]{2}{*}{ Dilatation } & Baseline & $1.12 \pm 0.33$ & $1.14 \pm 0.35$ & $1.20 \pm 0.40$ & 0.441 \\
\hline & 6 hours later & $2.66 \pm 0.83$ & $2.25 \pm 0.56$ & $3.17 \pm 0.72$ & $<0.001$ \\
\hline$P$-value & & $<0.001$ & $<0.001$ & $<0.001$ & \\
\hline \multicolumn{2}{|c|}{ Interval between intervention to active phase ( $\mathrm{hr}$ ) } & $8.03 \pm 2.57$ & $9.57 \pm 1.92$ & $5.74 \pm 2.63$ & $<0.001$ \\
\hline \multicolumn{2}{|c|}{ Interval between intervention to delivery (hr) } & $11.40 \pm 3.51$ & $13.12 \pm 2.81$ & $9.25 \pm 3.20$ & $<0.001$ \\
\hline \multicolumn{2}{|c|}{ Length of stay in hospital (day) } & $2.26 \pm 1.06$ & $1.88 \pm 0.82$ & $1.49 \pm 0.77$ & $<0.001$ \\
\hline \multirow[t]{2}{*}{ Type of delivery } & Vaginal & $39(60)$ & $46(70.8)$ & $53(81.5)$ & 0.026 \\
\hline & Cesarean & $26(40)$ & $19(29.2)$ & $12(18.5)$ & \\
\hline
\end{tabular}

Data are shown as mean \pm standard deviation or number (\%).

EASI, extra-amniotic saline infusion.

Table 3. Among-group comparisons according to ripening complications

\begin{tabular}{lcccc}
\hline & & Groups & EASI & \multicolumn{2}{c}{$\boldsymbol{P}$-value } \\
\cline { 2 - 4 } Complications & Misoprostol & Laminaria & $6(9.2)$ & 0.852 \\
\hline Dilatation arrest & $8(12.3)$ & $7(10.8)$ & $2(3.1)$ & 0.013 \\
Meconium staining & $11(16.9)$ & $1(6.2)$ & $1(1.5)$ & 0.352 \\
Chorioamnionitis & $2(3.1)$ & $3(4.6)$ & $2(3.1)$ & 0.016 \\
Abruptio placenta & $10(15.4)$ & $7(10.8)$ & $4(6.2)$ & 0.020 \\
Fetal distress & $14(21.5)$ & &
\end{tabular}

Values are presented as number (\%).

EASI, extra-amniotic saline infusion. 


\title{
Obstetrics \& Gynecology Science
}

\author{
Vol. 63, No. 3, 2020
}

parity, and BMI had not affected the study outcomes, including 6-hour Bishop scores and cervical dilatation (Table 4).

\section{Discussion}

This study compared the effects of vaginal misoprostol, laminaria, and EASI on cervical ripening and labor induction. Findings revealed that the time interval from labor induction to the labor active phase in the EASI group was significantly shorter than that in the laminaria and the misoprostol groups. Similarly, a previous study reported that this time interval was significantly shorter in the EASI group than in the laminaria group [8]. However, contrary to our findings, another study did not find any significant differences between the misoprostol and the laminaria groups regarding the time intervals between labor induction and the labor active phase, attributing this finding to the dose of misoprostol and repeat doses [10]. Another study also reported that the time interval between labor induction and the labor active phase in the misoprostol group was not significantly different from the EASI group probably due to the fact that the EASI catheter had been using traction [12]. In line with our findings, a study reported that the time interval between labor induction and the labor active phase in the misoprostol group was significantly shorter than that in the laminaria group [9]. However, contrary to our findings, another study showed that this time interval in the misoprostol group did not significantly differ from the EASI group [14]. The contradictory results between studies was attributable to misoprostol dosage differences.

Our findings also indicated that the time interval between labor induction and delivery in the EASI group was signifi- cantly shorter than that in the misoprostol and laminaria groups. In contrast, 2 studies showed that the misoprostol and EASI groups did not significantly differ from each other regarding the induction to delivery time intervals $[13,14]$, and several studies have reported significantly shorter induction to delivery time intervals in the misoprostol group than in the EASI group $[7,11,15]$. These contradictions may be due to the differences in misoprostol dosage among these studies. For instance, while misoprostol was administered as a single $25-\mu \mathrm{g}$ dose in the present study, it was administered as a single 100- $\mu$ g dose in 1 study [11] and as a 25- $\mu$ g dose repeated every 4 hours in another study [7]. Our findings also revealed that the induction to delivery time interval in the misoprostol group was significantly shorter than that in the laminaria group. A previous study reported the same findings [9], while another study reported no significant differences between these groups regarding the induction to delivery time intervals [10].

In the present study, the Bishop score increase in the EASI group was significantly greater than that in the other 2 groups. Contrary to this finding, some studies reported that the Bishop score increase in the misoprostol group was greater than that in either the EASI or laminaria group $[9,11,16]$. An explanation for this contradiction is the difference among the studies regarding misoprostol dosages.

We also found that 6 hours after labor induction, cervical dilation in the EASI group was significantly greater than that in the misoprostol and laminaria groups. In contrast, a study reported that cervical dilation 6 hours after induction in the laminaria group was 4.4-times greater than that in the misoprostol group [10], and another study did not find any significant difference between the laminaria and EASI groups regarding cervical dilation 6 hours after induction [8].

Table 4. Differences in 6-hour Bishop scores and cervical dilatation based on potential confounders

\begin{tabular}{|c|c|c|c|c|c|c|}
\hline \multirow{2}{*}{ Model } & \multicolumn{3}{|c|}{6 hours cervical dilatation } & \multicolumn{3}{|c|}{6 hours Bishop score } \\
\hline & Beta & $t$ & Significant & Beta & $t$ & Significant \\
\hline Constant & & 1.683 & 0.094 & & -0.799 & 0.425 \\
\hline Group & -0.443 & -7.718 & 0.000 & -0.331 & -5.855 & 0.000 \\
\hline Age & -0.014 & -0.228 & 0.820 & 0.085 & 1.479 & 0.141 \\
\hline Parity & 0.066 & 1.105 & 0.270 & 0.045 & 0.765 & 0.445 \\
\hline Gestational age & 0.029 & 0.506 & 0.613 & 0.148 & 2.648 & 0.009 \\
\hline BMI & -0.074 & -1.300 & 0.195 & -0.050 & -0.907 & 0.365 \\
\hline
\end{tabular}

BMI, body mass index. 


\section{Obstetrics \& Gynecology Science}

Zohreh Tabasi, et al. Misoprostol, laminaria, and EASI on cervical ripening

It appears that Foley catheters are more effective in causing cervical dilation, while misoprostol is more effective in changing cervical length and tone [17]. These facts can justify the greater effectiveness of mechanical cervical ripening methods (such as laminaria or transcervical catheters) in promoting cervical dilation.

The frequencies of cesarean sections in the EASI, misoprostol, and laminaria groups were $18.5 \%, 40 \%$, and $29.2 \%$, respectively, and the among group difference was statistically significant. Pairwise comparisons revealed that the frequency of cesarean sections in the EASI group was significantly less than that in the misoprostol group. However, there were no significant among group differences regarding the conditions which necessitated cesarean sections, including dilation arrest, uterine atony, and chorioamnionitis. In line with these findings, a study reported a higher prevalence of cesarean sections in the misoprostol group than in the EASI group [13]. Two other studies also reported that cesarean section rates in the misoprostol group were significantly greater than those in the laminaria group, though there were no significant between group differences regarding the indications for cesarean sections $[9,10]$. Moreover, in line with our findings, a previous study reported no significant differences between the laminaria and the trans-cervical groups regarding the routes of delivery [8]. The higher rate of cesarean sections in the misoprostol group is attributable to the fact that misoprostol can cause uterine hypertonicity and, thereby, increase the risk of fetal distress and the need for emergency cesarean sections. However, some studies reported lower rates of cesarean sections in the misoprostol group than in the EASI group $[7,16]$, and some studies found no significant difference between the misoprostol and the EASI groups regarding the rates of cesarean sections $[11,12,14]$. The difference among the studies regarding the rates of cesarean sections in the misoprostol, EASI, and laminaria groups may be due to the differences in the therapeutic protocols in different settings.

In the present study, the rates of placental abruption, fetal distress, and meconium staining in the misoprostol group were significantly greater than those in the other 2 groups. A previous study also reported that the rates of uterine hypertonicity and rupture in the misoprostol group were significantly higher than those in the EASI group. A higher uterine hypertonicity rate in the misoprostol group in that study was similar to our findings; however, there were no cases of uterine rupture in our study. This difference between the studies may be due to the high dose of misoprostol $(100 \mu \mathrm{g})$ in that study [11]. Contrary to our findings, an earlier study reported no significant difference between the misoprostol and laminaria groups regarding meconium staining [9]. Another study also reported that the rates of maternal and fetal consequences, such as uterine hypertonicity, uterine atony, and meconium staining, in the misoprostol group did not significantly differ from those in the EASI group [7]. However, a study found that the misoprostol and EASI groups differed significantly from each other regarding uterine hyperstimulation and tachysystole rates and did not differ from each other regarding meconium staining and chorioamnionitis rates [14]. Such differences among the studies are attributable to the differences in therapeutic care and patient management protocols in different healthcare settings.

Our findings also showed that the length of hospital stay was significantly shorter in the EASI group and significantly longer in the misoprostol group. These findings may be due to the higher rates of cesarean sections in the misoprostol group, which necessitated longer postoperative hospital stay. This variable has not been assessed in previous studies.

One of the limitations of this study was its non-blinded design because both the physicians and patients were aware of the study interventions. The other limitation was that we could not control the effects of psychological factors. These factors might have affected Bishop scores and labor progression. The strengths of the study were the large sample size (larger than that in previous studies) and the comparison of the 3 ripening methods. To the best of our knowledge, no previous study has compared the effects of the 3 ripening methods.

In conclusion, this study concluded that the EASI ripening method is more effective than misoprostol or laminaria in shortening the time intervals from labor induction to labor active phase and delivery. Moreover, the rates of cesarean sections and ripening complications which necessitated cesarean sections were significantly lower in the EASI group. Therefore, EASI, as a safe, inexpensive, and easily accessible method, is a better option than vaginal misoprostol and laminaria for cervical ripening and labor induction. More extensive studies are recommended to compare the effects of combined ripening methods such as combined misoprostol and EASI or combined laminaria and oxytocin. 


\section{Obstetrics \& Gynecology Science}

Vol. 63, No. 3, 2020

\section{Acknowledgements}

This study was derived from the doctoral thesis on obstetrics and gynecology and supported by the deputy of research, Kashan University of Medical Sciences (grant No. 97020). We also express our thanks to all women who made this study possible.

\section{Conflict of Interest}

No potential conflict of interest relevant to this article was reported.

\section{Ethical approval}

This study was approved by the Ethics Committee of Kashan University of Medical Sciences, Kashan, Iran (code: REC.1397.012) and registered in the Iranian Registry of Clinical Trial (code: IRCT20170513033941N39).

\section{Patient consent}

After receiving adequate information about the study methods and the potential complications of the 3 cervical ripening methods, participants signed the informed consent form for this study.

\section{References}

1. Kim HI, Choo SP, Han SW, Kim EH. Benefits and risks of induction of labor at 39 or more weeks in uncomplicated nulliparous women: a retrospective, observational study. Obstet Gynecol Sci 2019;62:19-26.

2. Association of Women's Health, Obstetric and Neonatal Nurses. Elective induction of labor. J Obstet Gynecol Neonatal Nurs 2019;48:227-9.

3. Cunningham FG, Leveno KJ, Bloom SL, Spong CY, Dashe JS, Hoffman BL, et al. Williams obstetrics. 25th ed. New York (NY): McGraw-Hill Education; 2018.

4. Pierce S, Bakker R, Myers DA, Edwards RK. Clinical insights for cervical ripening and labor induction using prostaglandins. AJP Rep 2018;8:e307-14.

5. Stephenson ML, Wing DA. A novel misoprostol delivery system for induction of labor: clinical utility and patient considerations. Drug Des Devel Ther 2015;9:2321-7.

6. Rath W, Kehl S. The renaissance of transcervical balloon catheters for cervical ripening and labour induction. Geburtshilfe Frauenheilkd 2015;75:1130-9.

7. Vahid Roudsari F, Ayati S, Ghasemi M, Hasanzadeh Mofrad M, Shakeri MT, Farshidi F, et al. Comparison of vaginal misoprostol with Foley catheter for cervical ripening and induction of labor. Iran J Pharm Res 2011;10:14954.

8. Zargar M, Nazari MA, Hormozy L, Madovi Mohammadi H. Efficacy of transcervical Foley catheter and laminaria on induction of labor in post-term pregnancy: a clinical study. Tehran Univ Med J 2014;72:161-6.

9. Souizi B, Mortazavi F, Haeri S, Borzoee F. Comparison of vaginal misoprostol, laminaria, and isosorbide dinitrate on cervical preparation and labor duration of term parturient: a randomized double-blind clinical trial. Electron Physician 2018;10:6756-63.

10. Behrashi M, Nezam-Zavareh J, Mesdaghinia E, Hashemi T, Mousavi GA, Mahdian M. Comparing the effects of vaginal misoprostol and laminaria on cervical ripening in labor induction among the term parturients. Feyz 2014;18:46-51.

11. Afolabi BB, Oyeneyin OL, Ogedengbe OK. Intravaginal misoprostol versus Foley catheter for cervical ripening and induction of labor. Int J Gynaecol Obstet 2005;89:263-7.

12. Fekrat M, Kashanian M, Hashem-Alavi S, Ali-Nezhad S. Comparing labor induction and cervical ripening methods including vaginal misoprostol, traction by Foley catheter and a combination of the two. J Reprod Infertil 2007;8:149-55.

13. Fox NS, Saltzman DH, Roman AS, Klauser CK, Moshier E, Rebarber A. Intravaginal misoprostol versus Foley catheter for labour induction: a meta-analysis. BJOG 2011;118:647-54.

14. Chung JH, Huang WH, Rumney PJ, Garite TJ, Nageotte MP. A prospective randomized controlled trial that compared misoprostol, Foley catheter, and combination misoprostol-Foley catheter for labor induction. Am J Obstet Gynecol 2003;189:1031-5.

15. Delaney S, Shaffer BL, Cheng YW, Vargas J, Sparks TN, 


\section{Obstetrics \& Gynecology Science}

Zohreh Tabasi, et al. Misoprostol, laminaria, and EASI on cervical ripening

Paul K, et al. Labor induction with a Foley balloon inflated to $30 \mathrm{~mL}$ compared with $60 \mathrm{~mL}$ : a randomized controlled trial. Obstet Gynecol 2010;115:1239-45.

16. Agarwal M, Kose V. Comparative study of vaginal misoprostol and intra cervical Foley's catheter for pre-induction cervical ripening at term. Int J Reprod Contracept
Obstet Gynecol 2017;6:20171008.

17. Adeniji AO, Olayemi O, Odukogbe AA. Intravaginal misoprostol versus transcervical Foley catheter in preinduction cervical ripening. Int J Gynaecol Obstet 2006;92:130-2. 